"die Elite" mitbesetzenden etablierten Parteien angekommen ist und er als demokratisches Korrektiv verstanden wird, stellt sich die Frage nach dem analytischen Gehalt des Begriffs. Dies gilt im Besonderen, wenn er selbst in der wissenschaftlichen Analyse eine politische Stoßrichtung erhält (Cuperus, S. 163 - 178). Populismus, also der anti-elitäre Gestus, ist für etablierte und regierungstragende Parteien stellenweise nützlich. Aber er ist nicht Markenkern von etablierten Parteien, die vielmehr eine Regierungsbeteiligung anstreben, öffentliche Ämter besetzen und staatliche Funktionen ausüben wollen. Der eingeführte Begriff Protestpartei als eine analytische Kategorie für Parteien, denen die Anti-Establishment-Symbolik im Gegensatz dazu als notwendiges Abgrenzungsmerkmal von der Funktionselite einer Gesellschaft dient, ist für diese Unterscheidung hilfreich.

Sebastian Rehse

\title{
21. Jahrbuch zu Extremismus und Demokratie: präzise Berichterstattung, inhaltsstarke Orientierung
}

Backes, Uwe, Alexander Gallus und Eckhard Jesse (Hrsg.): Jahrbuch Extremismus \& Demokratie 2009, Nomos Verlagsgesellschaft, Baden-Baden 2010, $€$ 49,-.

Zum ersten Mal von drei Herausgebern besorgt, liegt der 21. Band des Jahrbuches „Extremismus und Demokratie“ vor. Seine 504 Seiten gliedern sich in „Analysen“, „Daten, Dokumente, Dossiers“, einen 220 Seiten umfassenden Literaturteil sowie ein Editorial, „Mitteilungen und Hinweise“ und ergänzende Anhänge. Die beiden bisherigen Verantwortlichen sind als Herausgeber durch Alexander Gallus wirksam entlastet worden. Das Niveau der Publikation ist stabil, die Liste der Autoren beeindruckend. Neben etlichen anderen stehen die Namen Klaus von Beyme, Wilfried von Bredow, Hans Fenske, Iring Fetscher, Hannelore Horn, Peter Lösche, Wilfried Loth, Tilman Mayer, Werner Müller, Ursula Münch, Hiltrud Naßmacher, Ernst Nolte, Geoffrey K. Roberts, Alfons Söllner, Roland Sturm und Nikolaus Werz.

Inhaltlich setzt das Jahrbuch erfrischend „politically incorrect" mit einem Vergleich zwischen NPD und „Die Linke“ ein, wobei Eckhard Jesse bereits im Untertitel zwischen „einer harten und einer weichen Form des Extremismus" unterscheidet und damit den möglichen Vorwurf mangelnder Differenzierung antizipiert. Beiden untersuchten Parteien weist Jesse eine Gegnerschaft zur bestehenden freiheitlich-demokratischen Ordnung des Gemeinwesens nach, wobei die einen offensiv auftreten, die Parteiführung der anderen sich eher ambivalent verhält, verfassungsrevisionistischen Strömungen aber nicht entgegentritt (S. 13 - 31). Inhaltlich ergänzend erörtert Jürgen Lang, ob Die Linke als demokratische Partei klassifiziert werden könne. Lang sieht allenthalben Ambivalenzen durch ein Nebeneinander unaufgelöster Spannungen zu SED-Diktatur, Systemgegnern und elementaren Freiheitsrechten (S. 161 - 179). Der Rechtsextremismus wird von Elmar Vieregge durch eine biographische Skizze zu Andreas Molau, einem der wenigen Akademiker unter den Aktivisten, näher beleuchtet (S. 197 - 214). Präsentiert wird die berufliche Karriere von schriftstellerischen Anfängen über eine „bürgerliche“ Lehreretappe zu einer hauptamtlichen politischen Tätigkeit, die abrupt endet, als der Griff des Multifunktionärs nach dem NPD-Vorsitz 
misslingt, woraufhin er zur DVU wechselt. Nach der Fusion beider Parteien sind Vieregges Spekulationen über Molaus Chancen in der DVU unterdessen obsolet geworden.

In den Dokumentationen von Jesse (S. $101-114$ ) und Backes (S. 115-131) erscheint Die Linke als die dominierende Kraft jenseits von SPD und Bündnisgrünen, die in Westdeutschland durch ihre Fusion mit der WASG die früher isolierte Stellung der PDS hat überwinden können, so dass sie 2008 nur in Bayern den Einzug in das Landesparlament verfehlt hat. Backes hebt die Kommunistische Plattform und das trotzkistische Netzwerk „Marx21“ als personalpolitisch einflussreichste interne Strömungen hervor. Jesse arbeitet die elektoralen Größenunterschiede der Linken zu NPD und DVU heraus, die nicht einmal regelmäßig die Einprozentgrenze für die Wahlkampfkostenerstattung zu überwinden vermochten.

Alexander Gallus präsentiert durch einen Text der „Antifaschistischen Linken Berlin“ zur Luxemburg-Liebknecht-Ehrung die historische Dimension der Politik (S. 132 - 144). Zu recht betont er, dass Rosa Luxemburgs Diktum über die Freiheit der Andersdenkenden nur durch Uminterpretation in pluralistischem Sinne verstanden werden könne (S. 134).

Auf die aktuelle Kommunikation zielt Karsten D. Hoffmanns Beitrag über die Hamburger Szenezeitschrift "Zeck“, das erfolgreichste Blatt der Autonomenbewegung. Angesichts ihrer Bezugnahme auf Terroristen und politisch motivierte Gewalttaten hält Hoffmann den „zögerliche[n] Umgang staatlicher Organe“ mit der Zeitschrift für „verwunderlich“ (S. 239 $-256)$.

Mathias Brodkorb und Stefan Bruhn streben nach begrifflicher Klarheit für die Darstellung des rechten politischen Spektrums, wobei sie auch Kritik an den Herausgebern nicht scheuen (S. 145 - 160). Lazaros Miliopoulos sichtet neuere Totalitarismuskonzeptionen. Dazu unterscheidet er empirisch-vergleichende Konzepte von politiktheoretischen. Um den Begriff nach dem Zusammenbruch totalitärer Herrschaftsapparate von einer Bezugnahme auf diese frei zu halten, sucht Miliopoulos den Totalitarismus in „formalen Denkstrukturen und Zweckursachen“". Auf dieser Basis informiert er über Analyseobjekte und ungeklärte Fragen (S. $32-52)$.

Roland Sturm greift das Thema Nordirland auf (S. 53 - 71). Hinsichtlich der Bürgerkriegsparteien unterscheidet er zwischen „Unionisten“ und „Nationalisten“. Eine Analyse des Friedensprozesses mittels des konkordanzdemokratischen Konzepts Arend Lijpharts erweist sich als problematisch - bis hin zur Prognose Paul Dixons, die Befürworter der Konkordanz müssten ein Interesse an der Aufrechterhaltung der Konfrontation haben (S. 68). Aus einem Vergleich Nordirlands mit der Schweiz ergäbe sich wohl eine andere Prognose. Störend wirkt die Verkürzung der Sprache in den Spaltenüberschriften von Sturms Tabelle 1: „Überfälle Nationalisten“ und „Überfälle Unionisten“ lassen die Frage: "Wer wen?" unbeantwortet, da die Substantive ebenso durch „von“ wie durch „,auf" miteinander verbunden werden können.

Marianne Kneuers Porträt Spaniens zeigt die militanten baskisch-nationalistischen Gruppierungen als härteste Form des Extremismus im Lande, wohingegen die rechtsextremen Organisationen seit dem Ende der Diktatur marginalisiert, die linksextremen in langfristigem Niedergang befindlich sind. Schon die ehemalige Kommunistische Partei wird von Kneuer systemkonform interpretiert - ungeachtet ihrer autoritären inneren Führungsstrukturen unter Santiago Carrillo (S. 215 - 238).

In einem dritten auslandsorientierten Beitrag beleuchtet Michail Logvinov die Islamische Dschihad-Union (IJU), deren Wirksamkeit sich bis nach Deutschland erstreckt (S. 180 - 
196). Für nicht-islamische Länder können sich dabei mit Blick auf die IJU vor allem aus sozial nicht-integrierten, randständigen Gruppen potentielle Risiken ergeben.

Frank Decker setzt sich mit der Möglichkeit auseinander, auf Bundesebene plebiszitäre Instrumente in das deutsche Regierungssystem einzuführen (S. 72 - 97). Dabei argumentiert er ohne Differenzierungen zwischen „direkter Demokratie“ und „plebiszitärer Partizipation“, zwischen „Volk“ und „Stimmbürgerschaft" oder zwischen „pouvoir constituant" und „pouvoir constitué". Im Anschluss an Heidrun Abromeit werden weder Großbritannien noch die Schweiz als Verfassungsstaaten verstanden. Verfassungsstaatlichkeit steht dabei für Decker gegen Demokratie, wie sich in Deutschland am Bundesverfassungsgericht zeige, denn dieses lasse „das Demokratieprinzip (...) ins Leere laufen“. Schärfer noch: „Die Entdemokratisierung ist im verfassungsstaatlichen Konzept ausdrücklich intendiert" (S. 83). Das kann - und sollte - man mit Winfried Steffani und Martin Kriele wesentlich anders sehen. Auch Deckers Plädoyer für ein Bundesabstimmungssystem aufgrund einer angeblichen Heranführung der Bürger an repräsentative Institutionen und eines angeblich stärker konsensorientierten Politikstils (S. 95) vermag kaum zu überzeugen, denn die Wahlbeteiligung liegt in Systemen mit hoher Abstimmungsfrequenz generell niedrig und die Konsensorientierung in der Schweizer Politik beruht auf der Antizipation der unerwünschten Effekte von Referenden, nicht auf einer befriedenden Wirkung der Abstimmungen selbst - was sich an wiederkehrenden Themen eidgenössischer Abstimmungen leicht zeigen lässt. Widersprüchlich erscheinen schließlich die Ausführungen zu den Wirkungen von Abstimmungen auf das Parteiensystem: Behauptet Decker einmal „das nahezu vollständige Fehlen von Parteienkonkurrenz" in der Schweiz (S. 92), meint er später unter Hinweis auf FPÖ (Österreich) und SVP (Schweiz), „populistische oder extremistische Parteien“ könnten „aus der Möglichkeit des plebiszitären Agenda-settings im Wählerwettbewerb Vorteile ziehen" (S. 97). Für die historische deutsche Abstimmung über den Young-Plan (1929) soll dieser Vorteil aber aufgrund einer ohnehin wirksamen extremistischen Bedrohung irrelevant gewesen sein (S. 74). In erneutem Widerspruch hierzu behauptet Decker indes zum Schluss, plebiszitäre Elemente eigneten sich, um oppositionellen Protest in das System zu integrieren (S. 97). Der Abdruck des Beitrags dürfte danach vor allem die Liberalität der Herausgeber belegen.

Besonderen Wert legen diese auch auf den umfangreichen Literaturteil jedes Bandes. Die Breite der angezeigten und besprochenen Veröffentlichungen gibt dem Werk den Charakter einer umfassenden Dokumentation. Durch eine Alternativbesprechung von drei $\mathrm{Au}-$ toren, ambitionierte Sammelrezensionen und sorgfältige Einzelanalysen kommt dem Literaturteil zugleich orientierender Wert zu. Insgesamt ist damit erneut ein inhaltsstarker Band vorgelegt worden. 